\title{
Determination of Gas Well Productivity by Logging Parameters
}

\author{
Weijun $\mathrm{Hao}^{1}$, Zhihong Kang ${ }^{1,2} \&$ Dehua $\mathrm{Wu}^{1}$ \\ ${ }^{1}$ School of Energy Resources, China University of Geosciences(Beijing), China \\ ${ }^{2}$ Key Laboratory of Marine Reservoir Evolution and Hydrocarbon Accumulation Mechanism, Ministry of \\ Education, Beijing, China \\ Correspondence: Weijun Hao, School of Energy Resources, China University of Geosciences(Beijing), Beijing, \\ China. Tel: 86-189-1053-2151. E-mail: hwj199202@163.com
}

Received: March 24, 2017

Accepted: March 9, 2017

Online Published: April 25, 2017

doi:10.5539/esr.v6n2p56

URL: https://doi.org/10.5539/esr.v6n2p56

\begin{abstract}
The prediction and evaluation of reservoir productivity is a comprehensive index of the dynamic characteristics of gas reservoirs, which can provide a reasonable basis for the design and rational distribution of gasfield development plan. Proration of gas well is an important procedure in the development process, Absolute open flow as a key indicator of rational production of gas well. It is very important to determine the absolute open flow of the gas well. The Permian in Ordos Basin is a typical tight sandstone gas reservoir. The paper analyses correlation relations between different logging parameters and absolute open flow, and get the four parameters, porosity, permeability, storage coefficient (the product of porosity and effective thickness) with better correlation relations and effective thickness with best correlation relation by combining a large amount of gas logging data and static logging data and means of linear regression analysis, Then on the basis of this, a new empirical formula for calculating the absolute open flow of gas wells is obtained by using the method of multiple linear regression. The example shows that the result of this method is reasonable and reliable and the method can provide scientific basis for the prediction of natural gas absolute open flow of tight sandstone gas reservoirs.
\end{abstract}

Keywords: gas well productivity, logging parameters, multiple linear regression, tight sandstone gas reservoirs, Permian, absolute open flow

\section{Introduction}

Tight sandstone gas reservoir is an important component of unconventional oil and gas in our country at present, which has great exploration and development potential (Kang et al., 2016). Ordos Basin is a typical tight sandstone gas reservoir, the main river - delta facies sandstone, the main producing layer for Taiyuan, Shanxi and Shihezi group, reservoir average porosity is $7.8 \%$, the average permeability is $0.63 \mathrm{mD}$, The radius of the roar is less than $1 \mu \mathrm{m}$, the reservoir is characterized by low porosity and low permeability, heterogeneity is strong, production forecasting more difficult (Yang et al., 2015; Lu et al., 2015). The absolute open flow rate is considered as an important parameter to evaluate the productivity of gas wells. Generally the result is got through the system test, isochronal test, modified isochronal test, single point test and other productivity test method calculation ( $\mathrm{Lu}$ et al., 1998). The absolute open flow rate is an important parameter to evaluate the productivity of gas well. It is usually calculated by means of systematic well test, isochronal test, modified isochronal test, single point test and other productivity test methods (Gou, 2004, 2005). The production capacity test theory requires that the gas well test reach a stability condition. The production capacity test theory requires that the gas well test reach the relative stability condition. The productivity equation and the absolute open flow rate error of the heterogeneity low permeability gas well are determined, and the tight sandstone gas reservoir with low porosity and low permeability reaches the pressure stability. And the time required achieving stable pressure in tight sandstone reservoirs with low porosity and low permeability will take very long. System test, isochronal test, modified isochronal test are to be conducted at least four working systems of the production and pressure data test (Wang et al., 2010; Yang et al., 2012; Yan et al., 2013). The method will cost time and increase the economic cost. And different work system will inevitably affect the production of gas wells in the process.

The previous studies used the logging data to evaluate the gas well productivity semi-quantitative and quantitative evaluation methods (Shao et al., 2007). Gray correlation analysis is used to analyze the correlation and neural network method (Yan et al., 2007; Luo et al., 2006). Multivariate regression analysis was used to 
analyze the unrestricted flow, which was more convenient than the BP neural network, and could reflect the correlation between the parameters and the absolute open flow (Wang et al., 2006; Hu et al., 2010). In this paper, a large number of static logging data and dynamic test gas data are combined to determine the absolute open flow of gas wells by multiple linear regression method. The logging data is obtained before the formation is reconstructed, which can reflect the physical and objectivity of reservoir physical property and oil and gas content; and the test gas data can truly reflect the gas-bearing property of the reservoir. The method can reduce the operational risk, save the cost, improve the economic efficiency, and can be used as a new method to calculate the absolute open flow.

\section{Method}

\subsection{One-dimensional Linear Regression Analysis}

In order to establish an ideal model of absolute open flow forecasting, the selection of influencing factors is very important. In the Shenmu gas field of the Ordos Basin, the reservoir heterogeneity is strong and the reservoir fractures are not developed due to the interaction of sea-land alternation and digenesis. The main reservoir spaces are intergranular pores and dissolution pores. The Shenmu block develops the braided delta deposition, so the factors such as porosity and permeability, pore throat structure, gas saturation and thickness all have influence on gas well productivity (Cai et al., 2007). In this paper, the reservoir thickness, porosity, permeability, reservoir effective thicknesses are selected, and the storage capacity (the product of porosity and effective thickness) is introduced. The thickness and effective thickness of the reservoirs are important parameters in evaluating the reservoir, which are related to the reserves and abundance of the reservoir. When the porosity of the reservoir is the same, the bigger the porosity is, the stronger the seepage capacity is, and the porosity will directly influence the productivity of the gas well. The storage factor is the product of porosity and effective thickness and is an important indication of the ability of a well to contain the fluid. Permeability reflects the ability of fluid flow in the reservoir, which is an important factor affecting gas well productivity.

In order to analyze the influence of parameters on the absolute open flow, the Spearman correlation method is used to analyze the parameters. The Spearman rank correlation coefficient is a nonparametric property (independent of distribution) that measures the correlation between two variables, $\mathrm{X}$ and $\mathrm{Y}$, in the range $[-1,+1]$. The sign of the Spearman rank correlation coefficient represents the positive and negative correlations of the two variables $\mathrm{X}$ and $\mathrm{Y}$. If the Spearman rank correlation coefficient is positive, it indicates that $\mathrm{Y}$ increases as $\mathrm{X}$ increases, whereas $\mathrm{Y}$ decreases as $\mathrm{X}$ increases. When the Spearman rank correlation coefficient is 0 that means $\mathrm{Y}$ does not vary with $X$. The Spearman rank correlation coefficient is numerically close to \pm 1 indicating that the two variables $\mathrm{X}$ and $\mathrm{Y}$ are close to monotonic increasing or decreasing strictly. When the rank correlation coefficient is calculated, the data xi and yi are ordered from large to small, and the position of the data after ordering is defined as $a, b$, then $a$ and $b$ are the ranks of the variables $x i$ and yi, as di $=a-b$. When two same values are presented in the same column of data, the order of the two values is specified to be the same. According to the basic data analysis, if the data exist in the same rank, you should use the same rank correlation coefficient calculation method, the correlation coefficient is:

$$
\rho_{s}=\frac{\sum\left(\mathrm{x}_{i}-\bar{x}\right)\left(\mathrm{y}_{i}-\bar{y}\right)}{\sqrt{\sum\left(\mathrm{x}_{i}-\bar{x}\right)^{2} \sum\left(\mathrm{y}_{i}-\bar{y}\right)^{2}}}
$$

Cohen (1988) and other scholars put forward the correlation coefficient of the size of the criterion to determine the relevance of variables (Table 1)

Table 1. Criteria for judging relevance

\begin{tabular}{lll}
\hline Corelation & Negative Value & positive value \\
\hline uncorrelated & $-0.09 \sim 0.0$ & $0.0 \sim 0.09$ \\
low correlation & $-0.3 \sim-0.1$ & $0.1 \sim 0.3$ \\
Medium correlation & $-0.5 \sim-0.3$ & $0.3 \sim 0.5$ \\
significant correlation & $-1.0 \sim-0.5$ & $0.5 \sim 1.0$ \\
\hline
\end{tabular}

\subsection{Correlation Analysis of Absolute Open Flow}

According to the test data of Permian in the Ordos Basin, the data of wells in the Permian system in the 25 test gas results are selected (Table 2). Calculating reservoir thickness, effective thickness, porosity, storage capacity 
and permeability and absolute open flow of the Spearman correlation coefficient in the SPSS software and then analyze the Correlation of the parameters with the absolute open flow.

Table 2. Multiple Regression Analysis of the Basic Data

\begin{tabular}{lllllll}
\hline $\begin{array}{l}\text { Well } \\
\text { Number }\end{array}$ & $\begin{array}{l}\text { Reservoir } \\
\text { Thickness }(\mathrm{m})\end{array}$ & $\begin{array}{l}\text { Effective } \\
\text { Thickness } \\
(\mathrm{m})\end{array}$ & $\begin{array}{l}\text { Porosity } \\
(\%)\end{array}$ & $\begin{array}{l}\text { Storage } \\
\text { Coefficient } \\
\left(\Phi^{*} \mathrm{~h}\right)\end{array}$ & $\begin{array}{l}\text { Permeability } \\
(\mathrm{mD})\end{array}$ & $\begin{array}{l}\text { Absolute Open Fl } \\
\text { ow }\left(10^{4} \mathrm{~m}^{3} / \mathrm{d}\right)\end{array}$ \\
\hline 1 & 11.9 & 6.2 & 7.66 & 47.49 & 1.31 & 3.8018 \\
2 & 10.2 & 4.1 & 7.74 & 31.73 & 0.75 & 2.5399 \\
3 & 8.9 & 8.9 & 24.35 & 31.73 & 0.83 & 5.8489 \\
4 & 7.2 & 5.4 & 7.11 & 38.39 & 2.78 & 3.4036 \\
5 & 11.8 & 8.5 & 10.52 & 89.42 & 1.62 & 7.9283 \\
6 & 5.1 & 2.3 & 6.73 & 15.47 & 0.67 & 2.5031 \\
7 & 7.3 & 7.3 & 7.81 & 56.94 & 0.38 & 4.0454 \\
8 & 6.9 & 6.9 & 7.64 & 52.71 & 1.03 & 3.4367 \\
9 & 2.7 & 2.7 & 5.63 & 15.20 & 0.81 & 2.4582 \\
10 & 15.8 & 15.8 & 7.49 & 43.44 & 1.72 & 8.1154 \\
11 & 3.9 & 2.2 & 9.43 & 20.74 & 2.17 & 2.6108 \\
12 & 8.7 & 5.9 & 9.43 & 20.74 & 2.57 & 5.3948 \\
13 & 7.0 & 4.4 & 10.65 & 46.86 & 1.84 & 4.2067 \\
14 & 5.8 & 5.8 & 6.07 & 46.66 & 1.01 & 3.2245 \\
15 & 9.0 & 9.0 & 7.12 & 64.08 & 3.11 & 6.1441 \\
16 & 6.9 & 6.9 & 7.51 & 51.81 & 2.44 & 4.6321 \\
17 & 5.6 & 5.0 & 9.17 & 45.85 & 1.01 & 4.2818 \\
18 & 11.9 & 2.5 & 8.62 & 21.52 & 0.15 & 3.4929 \\
19 & 3.3 & 3.3 & 8.32 & 27.39 & 1.91 & 2.2009 \\
20 & 8.3 & 2.5 & 6.34 & 15.85 & 0.54 & 2.0976 \\
21 & 2.6 & 2.6 & 11.23 & 29.19 & 0.82 & 1.2173 \\
22 & 6.2 & 3.2 & 7.06 & 22.59 & 1.06 & 1.5486 \\
23 & 7.4 & 4.8 & 6.51 & 31.23 & 0.96 & 1.7535 \\
24 & 10.2 & 5.2 & 10.01 & 50.05 & 1.07 & 1.0012 \\
25 & 18.1 & 4.0 & 7.27 & 29.08 & 0.36 & 0.4355 \\
\hline
\end{tabular}

The results of the rank correlation coefficient of the calculated parameters and the absolute open flow rate are represented in Table 3.

Table 3. Rank correlation coefficient table

\begin{tabular}{llllll}
\hline Parameters & $\begin{array}{l}\text { Reservoir } \\
\text { Thickness }\end{array}$ & $\begin{array}{l}\text { Effective } \\
\text { Thickness }\end{array}$ & Porosity & Permeability & $\begin{array}{l}\text { Storage } \\
\text { Coefficient }\end{array}$ \\
\hline Absolute Open Flow & 0.27 & 0.782 & 0.301 & 0.324 & 0.486 \\
\hline
\end{tabular}

It can be seen from the table that the Spearman correlation coefficients of all factors and absolute open flow are positive, indicating a positive correlation with the absolute open flow. The effective thickness has a significant correlation with the absolute open flow, and the permeability, porosity and storage capacity are medium correlated. The correlation between the reservoir thickness and the absolute open flow is 0.27 , which is low correlation. The coefficient of correlation between the storage capacity and the absolute open flow is 0.486 , which is bigger than the correlation coefficient 0.31 between the porosity and the absolute open flow. The storage capacity is the product of the porosity and the effective thickness. The results shows that the combination of logging parameters in an appropriate way can improve the correlation with the absolute open flow.

Scatter plot analysis was performed on the reservoir thickness with the lowest correlation between absolute open flow and the highest effective reservoir thickness. The relationship between the reservoir thickness and the absolute open flow shows the data points are scattered and the tendency fitting is poor. The correlation coefficient R2 is 0.43 , indicating that the correlation degree is low. There is a positive correlation between the absolute open flow rate and the effective thickness, the correlation coefficient R2 is 0.74 , the value is close to 1 , 
and the correlation degree is high. Removing the reservoir thickness, choose four better parameters of the four correlation coefficients, and then use multiple linear regression method to establish an absolute open flow calculation model.

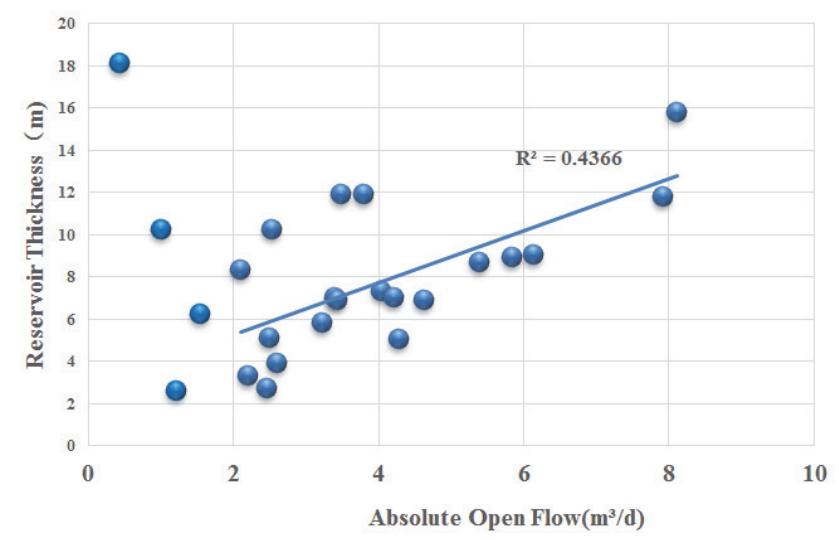

Figure 1. Catter diagram of reservoir thickness and absolute open flow

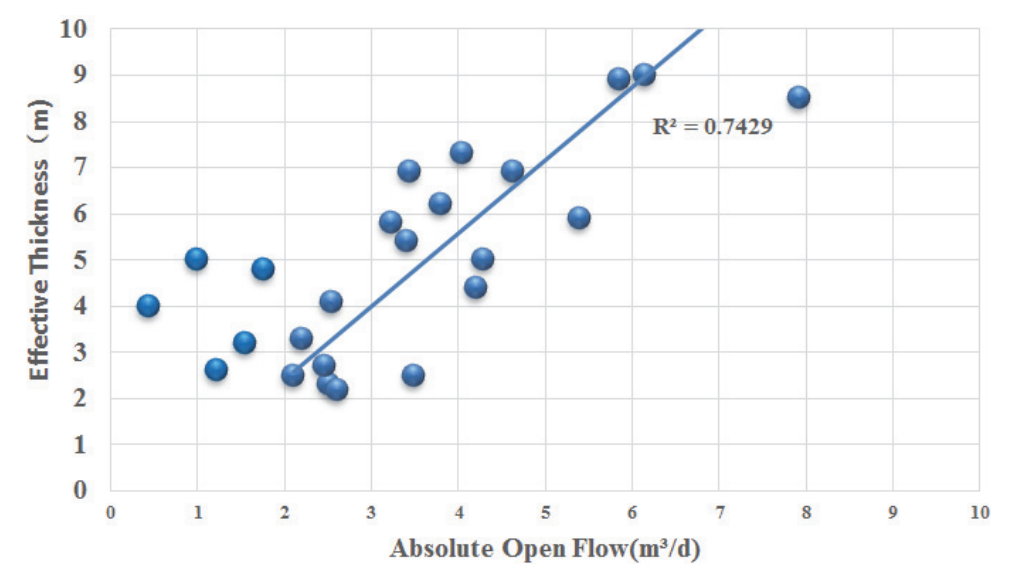

Figure 2. Cattering diagram of effective thickness and absolute open flow of gas layer

\subsection{Multiple Linear Regression Analysis Model}

The multiple linear regression equation is:

$$
y=\beta_{0}+\beta_{1} x_{1}+\beta_{2} x_{2}+\cdots+\beta_{k} x_{k}
$$

The regression coefficients in the multiple linear regressions can be estimated by the least squares method. Residual sum of squares is calculated as:

$$
S S E=\sum(y-\hat{y})^{2}=0
$$

$\hat{y}$ is the estimation of the sample. According to the principle of the minimum value calculation, we know that the residual sum of squares $S S E$ is a minimum. And when the partial derivatives of $S S E$ to $\beta_{0}, \beta_{1}, \cdots \beta_{k}$ are zero, $S S E$ takes the minimum value.

Make a partial derivation of $S S E$ and $\beta_{0}, \beta_{1}, \cdots \beta_{k}$, and make it equal to zero, we can gain a formulation $k+1$.

$$
\frac{\partial S S E}{\partial \beta_{i}}=-2 \sum(y-\hat{y})=0
$$




$$
\frac{\partial S S E}{\partial \beta_{0}}=-2 \sum(y-\hat{y}) x_{i}=0
$$

Multiple linear regression regression degree test needs to use the determination coefficient $R^{2}, R^{2}$ said independent variable dependent linear correlation degree, $0 \leq R^{2} \leq 1$, the value closer to 1 , the higher the regression equation fitting. It is defined as:

$$
R^{2}=\frac{S S R}{S S T}=1-\frac{S S E}{S S T}=1-\frac{\sum(y-\hat{y})^{2}}{\sum(y-\bar{y})^{2}}
$$

$S S R$ is the sum of squares of regressions, $S S E$ is the sum of squares of the residuals, and $S S T$ is the sum of squared deviations of the total deviation.

The size of the constant coefficient $R^{2}$ is affected by the number $x$ of the independent variable $k$. When the number of independent variables increases, it will cause the regression of the sum of squared $S S R$ increases, while $R^{2}$ increases. However, increasing the number of independent variables caused by $R^{2}$ increase can not represent the degree of fit, so by modifying $k$, to exclude the number of independent variables in the regression equation between the different degree of fitting, the number of independent variables to fit the excellent Degree of influence.

The modified method is: the original formulation of the numerator denominator, respectively, divided by their degrees of freedom, becomes the mean square error ratio, adjusted for $R^{2}$ :

$$
\bar{R}^{2}=1-\frac{S S E /(n-k-1)}{S S T /(n-1)}=1-\frac{S S E}{S S T} \cdot \frac{n-1}{n-k-1}=1-\left(1-R^{2}\right) \frac{n-1}{n-k-1}
$$

From $F$ statist the high degree of fit, $F$ statistic is more significant, $F$ statistic formulation is:

$$
F=\frac{R^{2} / k}{\left(1-R^{2}\right) /(n-k-1)}
$$

\section{Results}

When the number of independent variables is large, with the help of SPSS software, enter the appropriate dependent variable and independent variables, can be quickly calculated. Four parameters, such as effective thickness, storage capacity $\left(\Phi^{*} \mathrm{~h}\right)$ ), permeability and porosity, are input into the SPSS regression model in turn, and the following parameters are obtained.

Table 4. Model summary

\begin{tabular}{ccccc}
\hline Model & $\mathrm{R}$ & $\mathrm{R}^{2}$ & Adjusted $\mathrm{R}^{2}$ & Std. Error of the Estimate \\
\hline volue & 0.833 & 0.695 & 0.634 & 0.643 \\
\hline
\end{tabular}

$\mathrm{R}$ is a complex correlation coefficient, indicating that all independent variables in the model and the dependent variable between the linear regression relationship between the size of the larger $\mathrm{R}$ indicates that the more close to the linear regression relationship. The coefficient of determination $R^{2}$ is equal to 0.695 , the revised $R^{2}$ is 0.634 , the greater the value, the better the model fitting effects. The standard error reflects the accuracy of the model, the smaller the standard error, the better the fitting effect of the model. 
Table 5. Analysis of variance

\begin{tabular}{cccccc}
\hline Model & Sum of Squares & df & Mean Square & F & Sig. \\
\hline Regression & 65.999 & 4 & 16.500 & 11.377 & $.000^{\mathrm{a}}$ \\
Residual & 29.004 & 20 & 1.450 & & \\
Total & 95.003 & 24 & & & \\
\hline
\end{tabular}

The results of regression analysis are listed in the table: the sum of squares of regression, the degree of freedom of regression, the probability of significance, and so on. The probability of significant test of regression equation is 0 , less than the level of significance 0.05 , indicating that the coefficient is not equal to 0 at the same time, the explanatory variables and explanatory variables of the linear relationship is significant. A linear equation can be established with the model has statistical significance.

Table 6. Regression coefficient table

\begin{tabular}{|c|c|c|c|c|c|}
\hline \multirow{2}{*}{ Model } & \multicolumn{2}{|c|}{ Unstandardized Coefficients } & \multirow{2}{*}{$\begin{array}{l}\text { Standardized } \\
\text { Coefficients }\end{array}$} & \multirow{2}{*}{$\mathrm{t}$} & \multirow{2}{*}{ Sig. } \\
\hline & $\mathrm{B}$ & Std.Error & & & \\
\hline Constant & -0.127 & 0.689 & & -0.184 & 0.856 \\
\hline Effective Thickness & 0.409 & 0.111 & 0.621 & 3.680 & 0.001 \\
\hline Porosity & 0.006 & 0.006 & 0.132 & 0.981 & 0.005 \\
\hline Storage Coefficient & 0.020 & 0.018 & 0.179 & 1.134 & 0.027 \\
\hline Permeability & 0.446 & 0.355 & 0.166 & 1.258 & 0.036 \\
\hline
\end{tabular}

The unrestricted flow regression formulation obtained from the regression coefficient table:

Absolute open Flow $=-0.127+0.4093 \times$ Effective Thickness $+0.006 \times$ Porosity $+0.02 \times$ Storage Capacity + $0.446 \times$ Permeability

\section{Discussion}

In order to make sure the reliability and the range of error of the formulation, the tested gas and well logging data of 29 wells in the Ordos basin were selected as the tested data. The established empirical formulation was used to calculate the absolute open flow rate, and the results were compared with the productivity test Calculated results.

Table 7. Comparison of results of productivity test and model calculation

\begin{tabular}{cccccccc}
\hline $\begin{array}{c}\text { Well } \\
\text { Number }\end{array}$ & $\begin{array}{c}\text { Effective } \\
\text { Thickness } \\
(\mathrm{m})\end{array}$ & $\begin{array}{c}\text { Porosity } \\
(\%)\end{array}$ & $\begin{array}{c}\text { Storage } \\
\text { Coefficient } \\
(\Phi * \mathrm{~h})\end{array}$ & $\begin{array}{c}\text { Permeability } \\
(\mathrm{mD})\end{array}$ & $\begin{array}{c}\text { Productivity test } \\
\left(10^{4} \mathrm{~m}^{3} / \mathrm{d}\right)\end{array}$ & $\begin{array}{c}\text { Model } \\
\text { calculation } \\
\left(10^{4} \mathrm{~m}^{3} / \mathrm{d}\right)\end{array}$ & $\begin{array}{c}\text { Relative } \\
\text { Error }\end{array}$ \\
\hline 1 & 1.8 & 10.3 & 18.54 & 1.94 & 2.2044 & 1.9070 & 13.49 \\
2 & 15.4 & 8.2 & 123.35 & 1.18 & 11.4671 & 9.2130 & 19.66 \\
3 & 4.7 & 8.6 & 40.42 & 0.52 & 2.6536 & 2.8872 & 8.80 \\
4 & 4.2 & 8.6 & 36.12 & 0.15 & 3.4924 & 2.4317 & 30.32 \\
5 & 3.1 & 10.1 & 31.31 & 0.70 & 2.9287 & 2.1399 & 26.93 \\
6 & 11.5 & 10.7 & 123.05 & 5.13 & 11.6154 & 9.3897 & 19.12 \\
7 & 4.0 & 8.2 & 32.80 & 2.36 & 2.0152 & 3.2668 & 62.11 \\
8 & 21 & 5.7 & 119.70 & 0.79 & 12.9195 & 11.2425 & 12.98 \\
9 & 4.3 & 7.0 & 30.10 & 5.42 & 8.9775 & 4.6930 & 47.72 \\
10 & 15.5 & 6.8 & 105.40 & 1.02 & 10.2452 & 8.8162 & 13.95 \\
11 & 6.6 & 7.5 & 49.50 & 0.30 & 3.8263 & 3.7412 & 1.55 \\
12 & 3.5 & 8.2 & 28.70 & 0.98 & 2.0929 & 2.3648 & 12.99 \\
13 & 4.8 & 7.7 & 36.96 & 1.18 & 10.13263 & 3.1479 & 68.93 \\
14 & 4.1 & 7.7 & 31.57 & 0.75 & 2.5391 & 2.5620 & 0.91 \\
15 & 5.5 & 7.2 & 39.60 & 2.18 & 8.4808 & 3.9300 & 53.66 \\
16 & 5.2 & 7.1 & 36.92 & 2.78 & 3.4036 & 4.0207 & 18.13 \\
17 & 6.3 & 4.8 & 30.43 & 0.20 & 3.7385 & 3.1765 & 15.03 \\
18 & 1.8 & 7.8 & 14.06 & 1.94 & 2.2042 & 1.8025 & 18.22 \\
\hline
\end{tabular}




\begin{tabular}{lccccccc}
\hline 19 & 3.8 & 6.6 & 25.08 & 0.24 & 1.8384 & 2.0754 & 12.89 \\
20 & 16.0 & 5.7 & 91.20 & 0.76 & 12.9127 & 8.6142 & 33.28 \\
21 & 15.5 & 6.8 & 105.40 & 1.02 & 10.2429 & 8.8162 & 13.90 \\
22 & 4.7 & 7.9 & 37.13 & 0.23 & 3.2962 & 2.6879 & 18.30 \\
23 & 2.4 & 6.9 & 16.56 & 0.30 & 1.2453 & 1.3610 & 9.29 \\
24 & 4.0 & 9.3 & 37.20 & 1.10 & 2.4917 & 2.7994 & 12.35 \\
25 & 2.5 & 4.5 & 11.25 & 5.58 & 3.1278 & 3.6362 & 16.25 \\
26 & 6.3 & 9.1 & 57.27 & 0.35 & 6.4594 & 3.8057 & 41.08 \\
27 & 8.9 & 6.5 & 58.12 & 10.95 & 10.4438 & 9.5999 & 8.08 \\
28 & 6.4 & 5.4 & 34.30 & 0.48 & 3.3181 & 3.4229 & 3.16 \\
29 & 2.1 & 8.0 & 16.88 & 0.26 & 1.2453 & 1.2338 & 0.93 \\
\hline
\end{tabular}

To examine the accuracy of the model, a reasonable range of error is discussed. The range of relative error is less than $10 \%$, considering the simulation result is pretty good. The range of relative error is between $10 \%$ and $20 \%$, considering the simulation result is good. The range of relative error is between $20 \%$ and $30 \%$, considering the simulation result is in medium level. The range of relative error is more than $30 \%$ considering the simulation result is poor. The model is not very ideal. Analyze the table of the calculated result and presented in Table8

Table 8. Statistical results of model calculation

\begin{tabular}{cccc}
\hline Range of Relative Error & Model Effects & Well Number & The Proportion \\
\hline$<10 \%$ & pretty good & 7 & $24 \%$ \\
$10 \% \sim 20 \%$ & good & 14 & $48 \%$ \\
$20 \% \sim 30 \%$ & medium & 2 & $6.8 \%$ \\
$>30 \%$ & poor & 6 & $20.6 \%$ \\
\hline
\end{tabular}

By comparing the results of the model calculation with those of the productivity test results, the calculation accuracy of the model can be seen visually.

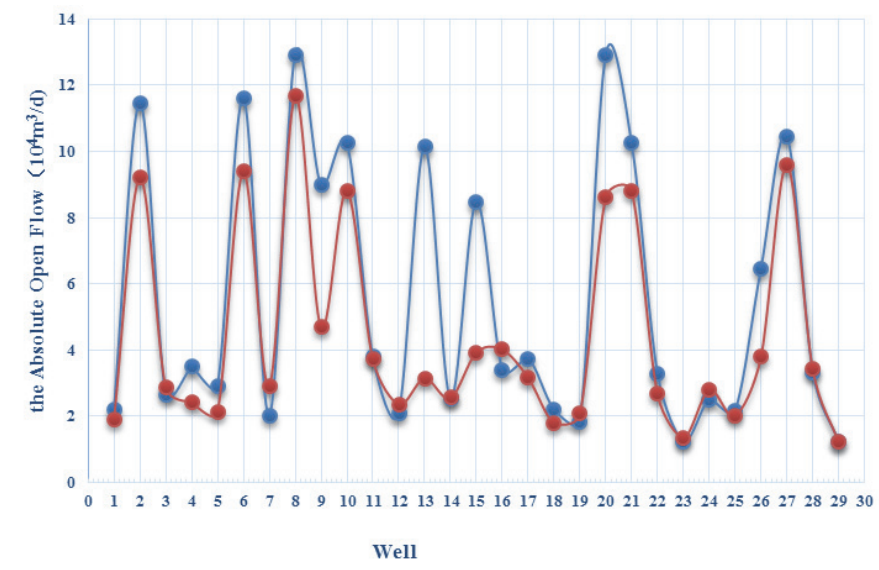

Figure 3. Comparison between model calculation results (red) and productivity test results(blue)

From the simulation result analysis table (Table 9), we can see that the established calculation model has high prediction accuracy. If the relative error is less than $20 \%$, it is considered that the simulation effect of the model is better. The calculated result of the empirical formulation is close to the absolute open flow value obtained by the productivity test and the coincidence rate is up to $72 \%$ degree. From the comparison of the calculated results (Fig 3), it can be seen that the obtained empirical formula has high prediction accuracy with the range of $2 \times 10^{4}$ $\sim 4 \times 10^{4} \mathrm{~m}^{3} / \mathrm{d}$, and the prediction precision is up to $90.9 \%$. The accuracy of the absolute open flow is larger than $8 \times 10^{4} \mathrm{~m}^{3} / \mathrm{d}$, but it is still within the acceptable range. According to previous studies, tight sandstone gas reservoirs need to be fractured and their productivity is affected by the half-length of fractures. As the length of fractures increases, the open-flow capacity of gas wells increases to a certain extent. The reasons for the large errors of some wells are analyzed, and the crack half-length explained by the pressure recovery test of the wells with irresistible flow of more than $8 \times 10^{4} \mathrm{~m}^{3} / \mathrm{d}$ and the wells with absolute open flow of less than $4 \times 10^{4} \mathrm{~m}^{3} / \mathrm{d}$ are 
also compared. And get the following table. It is found that the half-length of the fracture is larger than that of the smaller error, and the empirical formula is affected by the degree of fracturing. When the degree of fracturing is large, it will influence the calculation accuracy of empirical formulation

Table 9. Fracture half-length analysis table

\begin{tabular}{cccccc}
\hline Well & Fracture half-length $(\mathrm{m})$ & Relative Error $(\%)$ & Well & Fracture half-length $(\mathrm{m})$ & Relative Error $(\%)$ \\
\hline 7 & 60.5 & 62.11 & 23 & 13 & 9.29 \\
9 & 54.6 & 47.72 & 14 & 18.5 & 0.91 \\
15 & 42 & 53.66 & 11 & 23.8 & 1.55 \\
26 & 46.8 & 41.08 & 8 & 35.2 & 12.98 \\
\hline
\end{tabular}

Analyze the error, the following circumstances will cause the error becomes larger.

(1) The open mode of production layer. The fracturing thickness is less than the effective thickness and is not fully open.

(2) The effect of well heterogeneity. Parts of the stratigraphic litho logy mutation, sandstone pinch or local cracks are developed. Fracturing degree is too wide. Crack half-length is too wide, or crack width is too wide.

\section{Acknowledgments}

The multiple linear regression analysis of the Upper Paleozoic Permian series logging and well logging data in the eastern Ordos basin is used to obtain the new prediction method of the absolute open flow. The results are as follows:

(1) There is a positive correlation between the absolute open flow rate of gas well and the porosity, permeability, storage capacity and effective thickness of the reservoir. The correlation between the absolute open flow and the effective thickness is the largest, and the correlation between reservoir thickness and absolute open flow is low.

(2) In this paper, the correlation of effective thickness and the largest unrestricted flow is the largest. As a result, the definition of the effective thickness plays an important role of the calculation the accuracy of unrestricted flow.

(3) Combining logging parameters in an appropriate method can improve the correlation with absolute open flow, such as the correlation of the reservoir coefficient (the product of porosity to the effective thickness) to the absolute open flow rate is greater than the correlation between porosity and absolute open flow.

(4) The results present that the accuracy of the empirical formula is $76 \%$, which provides a new method for predicting the productivity of the Permian reservoir in the eastern Shenmu gas field. The new method has been applied to the prediction of reservoir productivity. The advantages are benefits for logging data calculation, saving time and improving economic efficiency. And it can be considered as an important non-stop supplement of production capacity test flow calculation.

(5) The obtained empirical formulation has high prediction accuracy in the range of $2 \times 10^{4} \sim 4 \times 10^{4} \mathrm{~m}^{3} / \mathrm{d}$, and the prediction precision is up to $90.9 \%$. The accuracy of the formulation is lower than that of the unconfined flow value of more than $8 \times 10^{4} \mathrm{~m}^{3} / \mathrm{d}$, and the accuracy of the formulation is affected by the degree of fracturing.

(6) Absolute open Flow $=-0.127+0.4093 \times$ Effective Thickness $+0.006 \times$ Porosity $+0.02 \times$ Storage Capacity $+0.446 \times$ Permeability, the method can provide scientific basis for the prediction of natural gas absolute open flow of tight sandstone gas reservoirs. At the same time, it provides a new method for predicting the productivity of tight sandstone, make full use of logging data, combined with multiple linear regression.

\section{References}

Gou, S. Q. (2005). Effects of heterogeneity on productivity testing of gas wells. Natural Gas Industry, 25(3), 109-112. http://dx.chinadoi.cn/10.3321\%2fj.issn\%3a1000-0976.2005.03.032

Hu, G. X., \& Gong, H. F. (2010). Application of Multiple Regression Analysis in Productivity Forecast for Low Permeability Reservoir. Gas Field Surface Engineering, 29(12), 23-25.

Li, H. Q., Liu, X. R., \& Ma, Z. G. (2012). Optimization Analysis of the Fracture Parameters of Horizontal Well of low permeability Gas Reservoir in Sulige. Journal of Yangtze University, 12(9), 92-95. http://dx.chinadoi.cn/10.3969\%2fj.issn.1673-1409(N).2012.12.030 
Lu, Tao, Liu, Y. X., \& Wu, L. C. et al. (2015). Challenges to and countermeasures for the production stabilization of tight sandstone gas reservoirs of the Sulige Gasfield, Ordos Basin. Natural Gas Industry, 35(6), 1-6. http://dx.chinadoi.cn/10.3787\%2fj.issn.1000-0976.2015.06.006

Luo, T. Y., \& Guo, J. C. (2006). Application of BP artificial nerve network to calculate absolute open flow of gas wells after fracturing. Journal of Southwest Petroleum University, 28(3), 65-69. http://dx.chinadoi.cn/10.3863\%2fj.issn.1674-5086.2006.03.019

Shao, W. Z., \& Wang, Z. Y. (2007). Research on productivity evalution in low porosity and permeability gas reservoir by logging data. Well Logging Technology, 31(6), 42-45. http://dx.chinadoi.cn/10.3969\%2fj.issn.1004-1338.2007.06.009

Sun, H. D. (2012). Modern Well Test Analysis and Production Evaluation in Complex Gas Reservoirs. Beijing: Petroleum Industry Press.

Wang, F. P., Huang, Q. H., \& Sun, L. (2010). A single point deliverability formula for gas well in low permeability gas reservoir. Xin Jiang Petroleum Geology, 31(6), 50-53.

Wang, J. G., \& He, S. L. (2006). New method on forecasting the absolute open-flow capacity of low permeability gas reservoir. Oil Drilling \& Production Teachnology, 28(6), 61-64. http://dx.chinadoi.cn/10.3969\%2fj.issn.1000-7393.2006.06.019

Yan, J. W., \& Zhang, S. Y. (2008). Relationship between reservoir log parameters and AOF in Daniudi gas field. Natural Gas Industry, 28(2), 67-69. http://dx.chinadoi.cn/10.3787\%2fj.issn.1000-0976.2008.02.017

Yan, N. P., Wang, X., \& Lv, H. (2013). Deliverability decline law of heterogeneous lower Paleozoic gas reservoirs in the Jingbian Gas Field, Ordos Basin. Natural Gas Industry, 33(2), 43-47. http://dx.chinadoi.cn/10.3787\%2fj.issn.1000-0976.2013.02.009

Yang, B., Fang, Y., \& Wang, G. Z. (2012). Analysis and prediction of the deliverability decline for gas well. $\begin{array}{lllll}\text { Journal of Southwest Petroleum University, } & 34(4), & 105-109 .\end{array}$ http://dx.chinadoi.cn/10.3863\%2fj.issn.1674-5086.2012.04.015

Yang, Hua, Liu, X. S., \& Xiong, Y. X. (2015). Yang Hui et al. The Shenmu Gas Field in the Ordos Basin: Its discovery and reservoir-forming geological characteristics. Natural Gas Industry, 35(6), 1-13. http://dx.chinadoi.cn/10.3787\%2fj.issn.1000-0976.2015.06.001

Zhu, K. Y., \& Zhou, L. (2016). The strategic thinking of unconventional petroleum and gas in China. Earth Science Frontiers, 23(2), 1-7. http://dx.chinadoi.cn/10.13745\%2fj.esf.2016.02.001

\section{Copyrights}

Copyright for this article is retained by the author(s), with first publication rights granted to the journal.

This is an open-access article distributed under the terms and conditions of the Creative Commons Attribution license (http://creativecommons.org/licenses/by/4.0/). 